\title{
TOP LOCAL COHOMOLOGY MODULES WITH SPECIFIED ATTACHED PRIMES
}

\author{
MOHAMMAD T. DIBAEI AND RAHELEH JAFARI
}

\begin{abstract}
Let $(R, \mathfrak{m})$ be a complete Noetherian local ring and let $M$ be a finite $R$-module of positive Krull dimension $n$. It is shown that any subset $T$ of $\operatorname{Assh}_{R}(M)$ can be expressed as the set of attached primes of the top local cohomology module $\mathrm{H}_{\mathfrak{a}}^{n}(M)$ for some ideal $\mathfrak{a}$ of $R$. Moreover if $\mathfrak{a}$ is an ideal of $R$ such that the set of attached primes of $\mathrm{H}_{\mathfrak{a}}^{n}(M)$ is a non-empty proper subset of $\operatorname{Assh}_{R}(M)$, then $\mathrm{H}_{\mathfrak{a}}^{n}(M) \cong \mathrm{H}_{\mathfrak{b}}^{n}(M)$ for some ideal $\mathfrak{b}$ of $R$ with $\operatorname{dim}_{R}(R / \mathfrak{b})=1$.
\end{abstract}

\section{INTRODUCTION}

Throughout $(R, \mathfrak{m})$ is a commutative Noetherian local ring with maximal ideal $\mathfrak{m}, M$ is a nonzero finite (i.e. finitely generated) $R$-module with positive Krull dimension $n:=\operatorname{dim}_{R}(M)$ and $\mathfrak{a}$ denotes an ideal of $R$. Recall that for an $R$-module $N$, a prime ideal $\mathfrak{p}$ of $R$ is said to be an attached prime of $N$, if $\mathfrak{p}=\operatorname{Ann}_{R}(N / K)$ for some submodule $K$ of $N$ (see [5]). The set of attached primes of $N$ is denoted by $\operatorname{Att}_{R}(N)$. If $N$ is an Artinian $R$-module so that $N$ admits a reduced secondary representation $N=N_{1}+\cdots+N_{r}$ such that $N_{i}$ is $\mathfrak{p}_{i}$-secondary, $i=1, \ldots, r$, then $\operatorname{Att}_{R}(N)=\left\{\mathfrak{p}_{1}, \ldots, \mathfrak{p}_{r}\right\}$ is a finite set.

Denote by $\mathrm{H}_{\mathfrak{a}}^{n}(M)$ the $n$th right derived functor of

$$
\Gamma_{\mathfrak{a}}(M)=\left\{x \in M \mid \mathfrak{a}^{r} x=0 \text { for some positive integer } r\right\}
$$

applied to $M$. It is well-known that $\mathrm{H}_{\mathfrak{a}}^{n}(M)$ is an Artinian module. Macdonald and Sharp, in [5], studied $\mathrm{H}_{\mathfrak{m}}^{n}(M)$ and showed that $\operatorname{Att}_{R}\left(\mathrm{H}_{\mathfrak{m}}^{n}(M)\right)=\operatorname{Assh}_{R}(M)$ where $\operatorname{Assh}_{R}(M):=\{\mathfrak{p} \in$ $\left.\operatorname{Ass}_{R}(M) \mid \operatorname{dim}_{R}(R / \mathfrak{p})=n\right\}$. It is shown in [4 Theorem A], that for any arbitrary ideal $\mathfrak{a}$ of $R, \operatorname{Att}_{R}\left(\mathrm{H}_{\mathfrak{a}}^{n}(M)\right)=\left\{\mathfrak{p} \in \operatorname{Ass}_{R}(M) \mid \mathrm{H}_{\mathfrak{a}}^{n}(R / \mathfrak{p}) \neq 0\right\}$ which is a subset of $\operatorname{Assh}_{R}(M)$. In [3], the structure of $\mathrm{H}_{\mathfrak{a}}^{n}(M)$ is studied by the first author and Yassemi and they showed that, in case $R$ is complete, for any pair of ideals $\mathfrak{a}$ and $\mathfrak{b}$ of $R$, if $\operatorname{Att}_{R}\left(\mathrm{H}_{\mathfrak{a}}^{n}(M)\right)=\operatorname{Att}_{R}\left(\mathrm{H}_{\mathfrak{b}}^{n}(M)\right)$, then $\mathrm{H}_{\mathfrak{a}}^{n}(M) \cong \mathrm{H}_{\mathfrak{b}}^{n}(M)$. They also raised the following question in [2] Question 2.9] which is the main object of this paper.

Question. For any subset $T$ of $\operatorname{Assh}_{R}(M)$, is there an ideal $\mathfrak{a}$ of $R$ such that $\operatorname{Att}_{R}\left(\mathrm{H}_{\mathfrak{a}}^{n}(M)\right)=T$ ?

This paper provides a positive answer for this question in the case $R$ is complete.

\section{Main Result}

In this section we assume that $R$ is complete with respect to the $\mathfrak{m}$-adic topology. As mentioned above, $\operatorname{Att}_{R}\left(\mathrm{H}_{\mathfrak{m}}^{n}(M)\right)=\operatorname{Assh}_{R}(M)$ and $\operatorname{Att}_{R}\left(\mathrm{H}_{R}^{n}(M)\right)=\emptyset$ is the empty set. Also $\operatorname{Att}_{R}\left(\mathrm{H}_{\mathfrak{a}}^{n}(M)\right) \subseteq \operatorname{Assh}_{R}(M)$ for all ideals $\mathfrak{a}$ of $R$. Our aim is to show that as $\mathfrak{a}$ varies over ideals of $R$, the set $\operatorname{Att}_{R}\left(\mathrm{H}_{\mathfrak{a}}^{n}(M)\right)$ takes all possible subsets of $\operatorname{Assh}_{R}(M)$ (see Theorem 2.8). In the following results we always assume that $T$ is a non-empty proper subset of $\operatorname{Assh}_{R}(M)$

In our first result we find a characterization for a subset of $\operatorname{Assh}_{R}(M)$ to be the set of attached primes of the top local cohomology of $M$ with respect to an ideal $\mathfrak{a}$.

2000 Mathematics Subject Classification. 13D45, 13D07.

Key words and phrases. Local cohomology, Associated primes, Attached primes The research of the first author was in part supported from IPM (No. 00000000). 
Proposition 2.1. Assume that $n:=\operatorname{dim}_{R}(M) \geq 1$ and that $T$ is a proper non-empty subset of Assh $_{R}(M)$. Set $\operatorname{Assh}_{R}(M) \backslash T=\left\{\mathfrak{q}_{1}, \ldots, \mathfrak{q}_{r}\right\}$. The following statements are equivalent.

(i) There exists an ideal $\mathfrak{a}$ of $R$ such that $\operatorname{Att}_{R}\left(H_{\mathfrak{a}}^{n}(M)\right)=T$.

(ii) For each $i, 1 \leq i \leq r$, there exists $Q_{i} \in \operatorname{Supp}_{R}(M)$ with $\operatorname{dim}_{R}\left(R / Q_{i}\right)=1$ such that

$$
\bigcap_{\mathfrak{p} \in T} \mathfrak{p} \nsubseteq Q_{i} \quad \text { and } \quad \mathfrak{q}_{i} \subseteq Q_{i} .
$$

With $Q_{i}, 1 \leq i \leq r$, as above, Att $_{R}\left(H_{\mathfrak{a}}^{n}(M)\right)=T$ where $\mathfrak{a}=\bigcap_{i=1}^{r} Q_{i}$.

Proof. $(i) \Rightarrow(i i)$. By [4] Theorem A], $\mathrm{H}_{\mathfrak{a}}^{n}(R / \mathfrak{p}) \neq 0$ for all $\mathfrak{p} \in T$, that is $\mathfrak{a}+\mathfrak{p}$ is $\mathfrak{m}$-primary for all $\mathfrak{p} \in T$ (by Lichtenbaum-Hartshorne Theorem). On the other hand, for $1 \leq i \leq r, \mathfrak{q}_{i} \notin T$ which is equivalent to say that $\mathfrak{a}+\mathfrak{q}_{i}$ is not an $\mathfrak{m}$-primary ideal. Hence there exists a prime ideal $Q_{i} \in \operatorname{Supp}_{R}(M)$ such that $\operatorname{dim}_{R}\left(R / Q_{i}\right)=1$ and $\mathfrak{a}+\mathfrak{q}_{i} \subseteq Q_{i}$. It follows that $\bigcap_{\mathfrak{p} \in T} \mathfrak{p} \nsubseteq \subseteq Q_{i}$.

$(i i) \Rightarrow(i)$. Set $\mathfrak{a}:=\bigcap_{i=1}^{r} Q_{i}$. For each $i, 1 \leq i \leq r, \mathfrak{a}+\mathfrak{q}_{i} \subseteq Q_{i}$ implies that $\mathfrak{a}+\mathfrak{q}_{i}$ is not $\mathfrak{m}$-primary and so $\mathrm{H}_{\mathfrak{a}}^{n}\left(R / \mathfrak{q}_{i}\right)=0$. Thus $\operatorname{Att}_{R} \mathrm{H}_{\mathfrak{a}}^{n}(M) \subseteq T$. Assume $\mathfrak{p} \in T$ and $Q \in \operatorname{Supp}(M)$ such that $\mathfrak{a}+\mathfrak{p} \subseteq Q$. Then $Q_{i} \subseteq Q$ for some $i, 1 \leq i \leq r$. Since $\mathfrak{p} \nsubseteq Q_{i}$, we have $Q_{i} \neq Q$, so $Q=\mathfrak{m}$. Hence $\mathfrak{a}+\mathfrak{p}$ is $\mathfrak{m}$-primary ideal. Now, by Lichtenbaum-Hartshorne Theorem, and by [4. Theorem A], it follows that $\mathfrak{p} \in \operatorname{Att}_{R}\left(\mathrm{H}_{\mathfrak{a}}^{n}(M)\right)$.

Corollary 2.2. If $H_{\mathfrak{a}}^{n}(M) \neq o$ then there is an ideal $\mathfrak{b}$ of $R$ such that $\operatorname{dim}_{R}(R / \mathfrak{b}) \leq 1$ and $H_{\mathfrak{a}}^{n}(M) \cong H_{\mathfrak{b}}^{n}(M)$.

Proof. If $\operatorname{Att}_{R}\left(\mathrm{H}_{\mathfrak{a}}^{n}(M)\right)=\operatorname{Assh}_{R}(M)$, then $\mathrm{H}_{\mathfrak{a}}^{n}(M)=\mathrm{H}_{\mathfrak{m}}^{n}(M)$. Otherwise $n \geq 1$ and $\operatorname{Att}_{R}\left(\mathrm{H}_{\mathfrak{a}}^{n}(M)\right)$ is a proper subset of $\operatorname{Assh}_{R}(M)$. Set $\operatorname{Assh}_{R}(M) \backslash \operatorname{Att}_{R}\left(\mathrm{H}_{\mathfrak{a}}^{n}(M)\right):=\left\{\mathfrak{q}_{1}, \cdots, \mathfrak{q}_{r}\right\}$. By Proposition 2.1, there are $Q_{i} \in \operatorname{Supp}_{R}(M)$ with $\operatorname{dim}_{R}\left(R / Q_{i}\right)=1, i=1, \cdots, r$, such that $\operatorname{Att}_{R}\left(\mathrm{H}_{\mathfrak{a}}^{n}(M)\right)=$ $\operatorname{Att}_{R}\left(\mathrm{H}_{\mathfrak{b}}^{n}(M)\right)$ with $\mathfrak{b}=\bigcap_{i=1}^{r} Q_{i}$. Now, by [3] Theorem 1.6], we have $\mathrm{H}_{\mathfrak{a}}^{n}(M) \cong \mathrm{H}_{\mathfrak{b}}^{n}(M)$. As $\operatorname{dim}(R / \mathfrak{b})=1$, the proof is complete.

Corollary 2.3. If $\operatorname{dim}_{R}(M)=1$ then any subset $T$ of $A s s h_{R}(M)$ is equal to the set $\operatorname{Att}_{R}\left(H_{\mathfrak{a}}^{1}(M)\right)$ for some ideal $\mathfrak{a}$ of $R$.

Proof. With notations as in Proposition 2.1, we take $Q_{i}=\mathfrak{q}_{i}$ for $i=1, \cdots, r$.

By a straightforward argument one may notice that the condition "complete" is superficial, for if $T$ is a non-empty proper subset of $\operatorname{Assh}_{R}(M)$, then $T=\operatorname{Att}_{R}\left(\mathrm{H}_{\mathfrak{a}}^{1}(M)\right)$, where $\mathfrak{a}=\underset{\mathfrak{p} \in \operatorname{Assh}_{R}(M) \backslash T}{\cap} \mathfrak{p}$.

The following is an example to Proposition 2.1.

Example 2.4. Set $R=k[[X, Y, Z, W]]$, where $k$ is a field and $X, Y, Z, W$ are independent indeterminates. Then $R$ is a complete Noetherian local ring with maximal ideal $\mathfrak{m}=(X, Y, Z, W)$. Consider prime ideals

$$
\mathfrak{p}_{1}=(X, Y) \quad, \quad \mathfrak{p}_{2}=(Z, W) \quad, \quad \mathfrak{p}_{3}=(Y, Z) \quad, \quad \mathfrak{p}_{4}=(X, W)
$$

and set $M=\frac{R}{\mathfrak{p}_{1} \mathfrak{p}_{2} \mathfrak{p}_{3} \mathfrak{p}_{4}}$ as an $R$-module, so that we have $\operatorname{Assh}_{R}(M)=\left\{\mathfrak{p}_{1}, \mathfrak{p}_{2}, \mathfrak{p}_{3}, \mathfrak{p}_{4}\right\}$ and $\operatorname{dim}_{R}(M)=2$. We get $\left\{\mathfrak{p}_{i}\right\}=\operatorname{Att}_{R}\left(H_{\mathfrak{a}_{i}}^{2}(M)\right)$, where $\mathfrak{a}_{1}=\mathfrak{p}_{2}, \mathfrak{a}_{2}=\mathfrak{p}_{1}, \mathfrak{a}_{3}=\mathfrak{p}_{4}, \mathfrak{a}_{4}=\mathfrak{p}_{3}$, and 
$\left\{\mathfrak{p}_{i}, \mathfrak{p}_{j}\right\}=\operatorname{Att}_{R}\left(H_{\mathfrak{a}_{i j}}^{2}(M)\right)$, where

$$
\begin{aligned}
& \mathfrak{a}_{12}=\left(Y^{2}+Y Z, Z^{2}+Y Z, X^{2}+X W, W^{2}+W X\right), \\
& \mathfrak{a}_{34}=\left(Z^{2}+Z W, X^{2}+Y X, Y^{2}+Y X, W^{2}+W Z\right), \\
& \mathfrak{a}_{13}=\left(Z^{2}+X Z, W^{2}+W Y, X^{2}+X Z\right), \\
& \mathfrak{a}_{14}=\left(W^{2}+W Y, Z^{2}+Z Y, Y^{2}+Y W\right), \\
& \mathfrak{a}_{23}=\left(X^{2}+X Z, Y^{2}+W Y, W^{2}+Z W\right), \\
& \mathfrak{a}_{24}=\left(X^{2}+X Z, Y^{2}+W Y, Z^{2}+Z W\right) .
\end{aligned}
$$

Finally, we have $\left\{\mathfrak{p}_{i}, \mathfrak{p}_{j}, \mathfrak{p}_{k}\right\}=A t t_{R}\left(H_{\mathfrak{a}_{i j k}}^{2}(M)\right)$, where $\mathfrak{a}_{123}=(X, W, Y+Z), \mathfrak{a}_{234}=(X, Y, W+Z)$, $\mathfrak{a}_{134}=(Z, W, Y+X)$.

Lemma 2.5. Assume that $n:=\operatorname{dim}_{R}(M) \geq 2$, and that $T$ is a non-empty subset of $A s s h_{R}(M)$ such that $\bigcap_{\mathfrak{p} \in T} \mathfrak{p} \nsubseteq \bigcap_{\mathfrak{q} \in A s s h_{R}\left(R / \sum_{\mathfrak{p} \in T^{\prime}} \mathfrak{p}\right)} \mathfrak{q}$, where $T^{\prime}=A s s h_{R}(M) \backslash T$. Then there exists a prime ideal $Q \in \operatorname{Supp}_{R}(M)$ with $\operatorname{dim}_{R}(R / Q)=1$ and $\operatorname{Att}_{R}\left(H_{Q}^{n}(M)\right)=T$.

Proof. Set $s:=\operatorname{ht}_{M}\left(\sum_{\mathfrak{p} \in T^{\prime}} \mathfrak{p}\right)$. We have $s \leq n-1$, otherwise $\operatorname{Assh}_{R}\left(R / \sum_{\mathfrak{p} \in T^{\prime}} \mathfrak{p}\right)=\{\mathfrak{m}\}$ which contra-

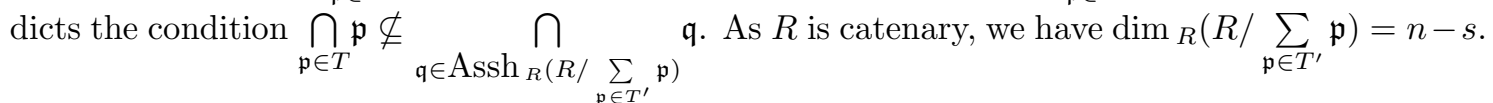
We first prove, by induction on $j, 0 \leq j \leq n-s-1$, that there exists a chain of prime ideals $Q_{0} \subset Q_{1} \subset \cdots \subset Q_{j} \subset \mathfrak{m}$ such that $Q_{0} \in \operatorname{Assh}_{R}\left(R / \sum_{\mathfrak{p} \in T^{\prime}} \mathfrak{p}\right), \operatorname{dim}_{R}\left(R / Q_{j}\right)=n-s-j$ and $\bigcap_{\mathfrak{p} \in T} \mathfrak{p} \nsubseteq Q_{j}$. There is $Q_{0} \in \operatorname{Assh}_{R}\left(R / \sum_{\mathfrak{p} \in T^{\prime}} \mathfrak{p}\right)$ such that $\bigcap_{\mathfrak{p} \in T} \mathfrak{p} \nsubseteq Q_{0}$. Note that $\operatorname{dim}_{R}\left(R / Q_{0}\right)=$ $\operatorname{dim}_{R}\left(R / \sum_{\mathfrak{p} \in T^{\prime}} \mathfrak{p}\right)=n-s$. Now, assume that $0<j \leq n-s-1$ and that we have proved the existence of a chain $Q_{0} \subset Q_{1} \subset \cdots \subset Q_{j-1}$ of prime ideals such that $Q_{0} \in \operatorname{Assh}_{R}\left(R / \sum_{\mathfrak{p} \in T^{\prime}} \mathfrak{p}\right)$, $\operatorname{dim}_{R}\left(R / Q_{j}\right)=n-s-(j-1)$ and that $\bigcap_{\mathfrak{p} \in T} \mathfrak{p} \nsubseteq Q_{j-1}$. Note that we have $n-s-(j-1)=$ $n-s+1-j \geq 2$. Therefore the set $V$ defined as

$$
V=\left\{\mathfrak{q} \in \operatorname{Supp}_{R}(M) \mid \begin{array}{ll}
Q_{j-1} \subset \mathfrak{q} \subset \mathfrak{q}^{\prime} \subseteq \mathfrak{m}, \operatorname{dim}_{R}(R / \mathfrak{q})=n-s-j, \\
& \left.\mathfrak{q}^{\prime} \in \operatorname{Spec}(R) \text { and } \operatorname{dim}_{R}\left(R / \mathfrak{q}^{\prime}\right)=n-s-j-1\right\}
\end{array}\right.
$$

is non-empty and so, by Ratliff's weak existence theorem [6] Theorem 31.2], is not finite. As $\bigcap_{\mathfrak{p} \in T} \mathfrak{p} \not Q_{j-1}$, we have $Q_{j-1} \subset Q_{j-1}+\bigcap_{\mathfrak{p} \in T} \mathfrak{p}$. If, for $\mathfrak{q} \in V, \bigcap_{\mathfrak{p} \in T} \mathfrak{p} \subseteq \mathfrak{q}$, then $\mathfrak{q}$ is a minimal prime of $Q_{j-1}+\bigcap_{\mathfrak{p} \in T} \mathfrak{p}$. As $V$ is an infinite set, there is $Q_{j} \in V$ such that $\bigcap_{\mathfrak{p} \in T} \mathfrak{p} \not \subseteq Q_{j}$. Thus the induction is complete. Now by taking $Q:=Q_{n-s-1}$ and by Proposition 2.1, the claim follows.

Corollary 2.6. Assume that $n:=\operatorname{dim}_{R}(M) \geq 2$ and $T$ is a non-empty subset of $A s s h_{R}(M)$ with $|T|=\left|\operatorname{Assh}_{R}(M)\right|-1$. Then there is an ideal $\mathfrak{a}$ of $R$ such that $\operatorname{Att}_{R}\left(H_{\mathfrak{a}}^{n}(M)\right)=T$.

Proof. Note that $\operatorname{Assh}_{R}(M) \backslash T$ is a singleton set $\{\mathfrak{q}\}$, say, and so ht ${ }_{M}(\mathfrak{q})=0$ and $\bigcap_{\mathfrak{p} \in T} \mathfrak{p} \nsubseteq \mathfrak{q}$. Therefore, by Lemma 2.5, the result follows.

Lemma 2.7. Assume that $n:=\operatorname{dim}_{R}(M) \geq 2$ and $\mathfrak{a}_{1}$ and $\mathfrak{a}_{2}$ are ideals of $R$. Then there exists an ideal $\mathfrak{b}$ of $R$ such that $\operatorname{Att}_{R}\left(H_{\mathfrak{b}}^{n}(M)\right)=\operatorname{Att}_{R}\left(H_{\mathfrak{a}_{1}}^{n}(M)\right) \cap \operatorname{Att}_{R}\left(H_{\mathfrak{a}_{2}}^{n}(M)\right)$.

Proof. Set $T_{1}=\operatorname{Att}_{R}\left(\mathrm{H}_{\mathfrak{a}_{1}}^{n}(M)\right)$ and $T_{2}=\operatorname{Att}_{R}\left(\mathrm{H}_{\mathfrak{a}_{2}}^{n}(M)\right)$. We may assume that $T_{1} \bigcap T_{2}$ is a non-empty proper subset of $\operatorname{Assh}_{R}(M)$. Assume that $\mathfrak{q} \in \operatorname{Assh}_{R}(M) \backslash\left(T_{1} \cap T_{2}\right)=\left(\operatorname{Assh}_{R}(M) \backslash\right.$ 
$\left.T_{1}\right) \bigcup\left(\operatorname{Assh}_{R}(M) \backslash T_{2}\right)$. By Proposition 2.1, there exists $Q \in \operatorname{Supp}_{R}(M)$ with $\operatorname{dim}_{R}(R / Q)=1$ such that $\mathfrak{q} \subseteq Q$ and $\bigcap_{\mathfrak{p} \in T_{1} \cap T_{2}} \mathfrak{p} \nsubseteq Q$. Now, by Proposition 2.1, again there exists an ideal $\mathfrak{b}$ of $R$ such that $\operatorname{Att}_{R}\left(\mathrm{H}_{\mathfrak{b}}^{n}(M)\right)=T_{1} \bigcap T_{2}$.

Now we are ready to present our main result.

Theorem 2.8. Assume that $T \subseteq$ Assh $_{R}(M)$, then there exists an ideal $\mathfrak{a}$ of $R$ such that $T=$ $\operatorname{Att}_{R}\left(H_{\mathfrak{a}}^{n}(M)\right)$.

Proof. By Corollary 2.3, we may assume that $\operatorname{dim}_{R}(M) \geq 2$ and that $T$ is a non-empty proper subset of $\operatorname{Assh}_{R}(M)$. Set $T=\left\{\mathfrak{p}_{1}, \ldots, \mathfrak{p}_{t}\right\}$ and $\operatorname{Assh}_{R}(M) \backslash T=\left\{\mathfrak{p}_{t+1}, \ldots, \mathfrak{p}_{t+r}\right\}$. We use induction on $r$. For $r=1$, Corollary 2.6 proves the first step of induction. Assume that $r>1$ and that the case $r-1$ is proved. Set $T_{1}=\left\{\mathfrak{p}_{1}, \ldots, \mathfrak{p}_{t}, \mathfrak{p}_{t+1}\right\}$ and $T_{2}=\left\{\mathfrak{p}_{1}, \ldots, \mathfrak{p}_{t}, \mathfrak{p}_{t+2}\right\}$. By induction assumption there exist ideals $\mathfrak{a}_{1}$ and $\mathfrak{a}_{2}$ of $R$ such that $T_{1}=\operatorname{Att}_{R}\left(\mathrm{H}_{\mathfrak{a}_{1}}^{n}(M)\right)$ and $T_{2}=\operatorname{Att}_{R}\left(\mathrm{H}_{\mathfrak{a}_{2}}^{n}(M)\right)$. Now by the Lemma 2.7 there exists an ideal $\mathfrak{a}$ of $R$ such that $T=T_{1} \bigcap T_{2}=\operatorname{Att}_{R}\left(\mathrm{H}_{\mathfrak{a}}^{n}(M)\right)$.

Corollary 2.9. (See [1, Corollary 1.7]) With the notations as in Theorem 2.8, the number of non-isomorphic top local cohomology modules of $M$ with respect to all ideals of $R$ is equal to $2^{\left|A s s h_{R}(M)\right|}$.

Proof. It follows from Theorem 2.8 and [3] Theorem 1.6].

Acknowledgment. The authors would like to thank the referee for her/his comments.

\section{REFERENCES}

1. F. W. Call, On local cohomology modules, J. Pure Appl. Algebra 43 (1986), no. 2, 111-117.

2. M. T. Dibaei and S. Yassemi, Some regidity results for highest order local cohomology modules, Algebra Colloq., to appear.

3. M. T. Dibaei and S. Yassemi, Top local cohomology modules, Algebra Colloq., to appear.

4. M. T. Dibaei and S. Yassemi, Attached primes of the top local cohomology modules with respect to an ideal, Arch. Math. (Basel) 84 (2005), no. 4, 292-297.

5. I. G. Macdonald and R. Y. Sharp, An elementary proof of the non-vanishing of certain local cohomology modules, Quart. J. Math. Oxford 23 (1972), 197-204.

6. H. Matsumura, Commutative ring theory, Cambridge Studies in Advanced Mathematics, 8. Cambridge University Press, Cambridge, 1986.

Mohammad T. Dibaei, Faculty of Mathematical Sciences, Teacher Training University, Tehran, Iran, and Institute for Theoretical Physics and Mathematics (IPM), Tehran, Iran.

E-mail address: dibaeimt@ipm.ir

Raheleh Jafari, Faculty of Mathematical Sciences, Teacher Training University, Tehran, Iran

E-mail address: jafarirahele@yahoo.com 